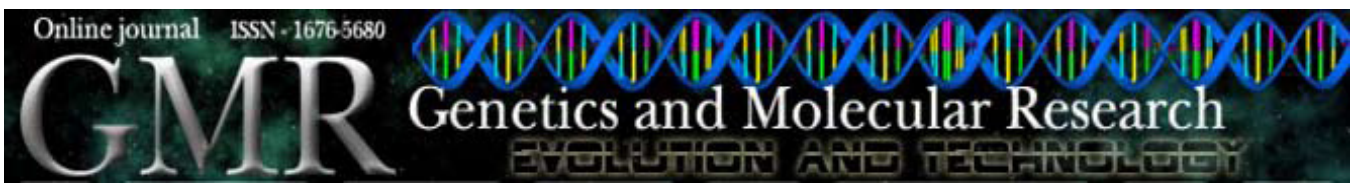

Thesis Abstract

\title{
Cytochemical and ultrastructural study of salivary glands of Triatomines (Heteroptera, Triatominae)
}

\author{
Ana Carolina Borella Anhê \\ 2007. Universidade Estadual Paulista, Instituto de Biociências, Letras e Ciências \\ Exatas (UNESP/IBILCE), São José do Rio Preto, SP, Brasil. Ph.D. thesis. 129p. \\ Orienting Professor: Dr. Maria Tercília Vilela de Azeredo-Oliveira.
}

The triatomines (Heteroptera, Reduviidae) are hematophagous insects commonly known as the "kissing bug", because they bite the uncovered face of sleeping people. The Triatominae subfamily is of medical/health importance, since these insects are transmitters of the protozoan Trypanosoma cruzi, etiologic agent of Chagas disease. This illness occurs mainly in South and Central America where, nowadays, it infects about 12 million people. Triatomines are also transmitters of Trypanosoma rangeli, which develops in their salivary glands, and although it is not pathogenic to humans, it causes mixed infections with T. cruzi, making differential diagnosis of the parasitemia difficult. Triatomines of the genera Triatoma and Panstrongylus have a complex gland composed of three pairs of salivary glands: the anterior (D1), median (D2) and posterior (D3); in the genus Rhodnius, there are just two pairs of glands: principal and accessory. The cells show intense synthesis of substances and have bulky nuclei with marked polyploidy. Blood-feeding insect saliva abounds in substances that antagonize the hemostatic, inflammatory and immunologic systems of the vertebrate host. The salivary glands contain phosphatases, which are responsible for the hydrolysis of organic phosphate esters and whose activity has been detected mainly in the nuclei of a great variety of animal and plant tissues. The present study was carried out with the genera Triatoma, Panstrongylus and Rhodnius in order to extend our understanding of the morphological, physiological and ultrastructural characteristics of their salivary glands, and to investigate the distribution of acid phosphatase and $\mathrm{Mg}^{2+}$-ATPase activities in this organ. The salivary glands were submitted to cytochemical assays, and the results showed that in Triatoma infestans and Panstrongylus megistus there was predominance of binucleated cells in D1 and D2 glands, and mononucleated ones in D3, and that in Rhodnius (R. neglectus and R. prolixus), there were just binucleated cells present. All salivary glands showed the presence of a bulky and polyploid nucleus, a clear association between nucleolar and heterochromatic bodies, cytoplasmic metachromasia and many secretory vesicles in the cytoplasm. Such characteristics were associated with intense biosynthetic activity to produce the secretions, essential 
for the hematophagous habit of triatomines. Acid phosphatase activity, evidenced in the same species, was especially detected in the nucleolus. Thus, the activity of this phosphatase during rDNA transcription, possibly in the nucleolar fibrillar center, is suggested. $\mathrm{Mg}^{2+}$-ATPase activity was evidenced in the salivary gland cells of T. infestans and P. megistus species. ATPase activity was detected, particularly in the euchromatin and nucleolus, where this enzyme probably acts in the transcription process. ATPase reaction was also evidenced in the nuclear membrane, which is associated with nuclear-cytoplasmic transport. Scanning electron microscopy contributed to salivary gland morphological characterization of Rhodnius genus ( $R$. neglectus and $R$. prolixus). It was demonstrated that the salivary gland complex is formed by two pairs of glands (principal and accessory) and ducts (principal and accessory), covered by tracheae (for oxygenation) and muscle fibers (whose contraction helps saliva secretion during the blood meal). 